\title{
Anaesthesia risks for non-cardiac procedures in cardiac patients
}

\author{
A Schure \\ Department of Anaesthesiology, Critical Care \& Pain Medicine, Boston Children's Hospital, United States of America \\ Corresponding author, email: annette.schure@childrens.harvard.edu
}

\section{Summary}

The number of patients with complex congenital heart disease (CHD) presenting for non-cardiac procedures is steadily increasing and more and more anaesthesiologists will be asked to participate in their care. This can be a very challenging task and will require adequate planning and preparation, but also some basic scientific inquiries.

Keywords: anaesthesia risks, complex congenital heart disease, non-cardiac procedures, cardiac patients

\section{The "5 W/H" approach:}

1. Why are we so worried?

2. When should we be worried?

3. Where should we do these procedures?

4. Who should do these patients?

5. What/How can we prepare for the future?

\section{Why are we so worried about these patients?}

Why: because there is plenty of evidence from our paediatric anaesthesia literature that cardiac patients have an increased risk for morbidity and mortality during anaesthesia. For example, according to the data from the Paediatric Cardiac Arrest Registry' a third of the reported arrests occurred in cardiac patients, mostly in infants and young children and in the general operating rooms. In Australia, a large tertiary paediatric hospital reported that in $50 \%$ of the anaesthesia related deaths pulmonary hypertension was the underlying medical problem. ${ }^{2}$

In addition to voluntary reports and single institution experiences, several recent big data studies investigated this patient population and confirmed the findings. Sulkowski and his group analysed an administrative database of 22 freestanding paediatric hospitals within a large network, looking at the frequency and type of non-cardiac surgeries in cardiac patients. They found that $41 \%$ patients who had cardiac surgery within the first year of life, had non-cardiac surgery by the age five years, over $50 \%$ in general surgery or otolaryngology. ${ }^{3}$

Another big data study used the 2012 paediatric database of the American College of Surgeons National Surgical Quality Improvement Program (ACS NSQIP Paediatric). Out of 51008 children in this database, $8.8 \%$ (4 520) were patients with CHD undergoing non-cardiac procedures. In a propensity-matched cohort study, the authors examined the differences in postoperative outcomes between children with and without CHD. Within the ACS NSQIP Paediatric database, the severity of the cardiac condition is classified into minor, major or severe CHD, based on residual lesion burden and functional status. The authors found a significantly increased odds ratio for overall mortality and need for re-intubation for the major and severe CHD groups. Patients with severe CHD were 7-8 times more likely to die after a procedure than non-cardiac patients. ${ }^{4}$

But what about specific procedures? Laparoscopic surgery, for example, a technique which has increasingly replaced many conventional surgical approaches.

David Chu and his group looked at the 2013/2014 data from the ACS NSQIP Paediatric database to evaluate the outcomes after laparoscopic surgery in children with and without congenital heart disease. Using the same CHD classification, they found that there was a significantly higher morbidity and mortality for major and severe CHD, and even minor CHD was associated with an increased 30-day morbidity. ${ }^{5}$

In a follow-up study, Chu and his colleagues repeated the analysis with an expanded database (2013-2015), this time comparing laparoscopic and open surgery. Given the complicated matching process and the limited number of patients, they were only able to report a "p for trend", but it seems that laparoscopic surgery is associated with lower morbidity and mortality for minor CHD, but this advantage is lost for more severe forms of CHD. Complex $\mathrm{CHD}$ patients have worse outcomes, independent of the surgical technique. And they do have an increased risk for readmission after laparoscopic surgery. ${ }^{6}$

What about day surgery, the other side of the surgical spectrum: Are cardiac patients eligible for ambulatory surgery? Is it safe?

In a large retrospective chart review of all anaesthetics over a 5 -year period at a single institution (Boston Children's Hospital) Yuki et al. found that, following a special screening process, $30 \%$ of the cardiac patients who presented for non-cardiac surgery underwent ambulatory procedures. The unplanned admission rate was $2.7 \%$, compared to $0.6-2.2 \%$ described in the paediatric literature. Cardiovascular events occurred in $3.9 \%$, (mainly the need for inotropic support), respiratory events in $1.8 \%{ }^{7}$ 
From a cost-oriented perspective, Faraoni and his group analysed the 2012 Kid's Inpatient Database: Children with CHD represented $4.4 \%$ of the total hospital discharges but accounted for $23 \%$ of the costs. $8 \%$ of the cardiac costs was attributed to non-cardiac surgery. ${ }^{8}$

Do we have additional information for the most complex CHD, the single ventricle patients?

In a small chart review study looking at 73 procedures in 40 patients with hypoplastic left heart syndrome (HLHS), a group from Ann Arbor found adverse events in 15\% and the need for ICU admission in $18 \%$. The majority of the procedures were done after the Glenn and Fontan. ${ }^{9}$

Scott Watkins and his group from Vanderbilt specifically investigated the stage I patients with HLHS who underwent open fundoplication and gastrostomy at their institution and noticed that perioperative instability was very common, as well as postoperative escalation of haemodynamic and respiratory support. ${ }^{10}$

For Boston Children's hospital, Morgan Brown reviewed the charts of single ventricle patients from 2007-2014 for the frequency and complication rate of non-cardiac surgery: $16.7 \%$ underwent non-cardiac surgery before their second stage palliation. The most common procedures were line insertions, G-tube placements and airway evaluations. Overall the perioperative rate of adverse events was $11.8 \%$ and included arrhythmias, haemodynamic and respiratory problems as well as cardiac arrest. ${ }^{11}$

\section{When should we be worried?}

With plenty of evidence that complex cardiac patients are at higher risk during non-cardiac procedures, the question remains how to define high-risk patients. The ASA classification is widely used by many specialties and has a long history, but it is highly subjective and varies significantly between individual users. RACHS-1-and the ACS NSQUIP classifications, based on surgical complexity, mortality, residual lesion burden and functional status, are very broad and do not consider additional conditions and procedural factors. ${ }^{12}$

White and his British colleagues published a review article in 2012 , using a combination of underlying pathophysiology, type of lesion, planned surgery and urgency as well as age and physical status to create three different risk categories (high, intermediate and low) with specific management recommendations. ${ }^{13}$

Faraoni's group analysed the 2012 and 2013 data from the ACS NSQIP paediatric database and identified eight preoperative predictors for in-hospital mortality which they developed into a risk score and validated with the 2014 data. The eight predictors were: emergency procedure, severe CHD, single ventricle physiology, surgery within 30 days, preoperative CPR and other important markers of critical illness (e.g. inotropic support, renal dysfunction and mechanical ventilation). A score of seven or more was associated with a high risk and an in-house mortality of $15-50 \% .^{14}$
Scoring systems like these will have to be validated in future studies but can be very helpful for planning and resource allocation.

\section{Where should we do these procedures?}

Where should cardiac patients go for their non-cardiac procedures? Should they always be referred to the highly specialised tertiary centre with cardiac expertise to optimise the safety or are there patients and procedures that could be done at the local level to minimise the burden for the families? And who makes the decision?

Currently, we witness an increasing centralisation of paediatric subspecialties in verified level I facilities and less and less paediatric experience at the local level. ${ }^{15} \mathrm{~A}$ trend that emphasises safety, but also comes at a price: A centralised expert care will concentrate the paediatric specialty workforce in urban areas, possibly impair the timely access to quality care in rural areas, especially for emergencies; and will invariably widen the gap in healthcare distribution. ${ }^{16}$

In the UK, where the national healthcare system has already restricted the number of paediatric cardiac centres, a recent publication by a non-cardiac tertiary paediatric centre tried to demonstrate that, with adequate communication and planning, comparable mortality and morbidity rates can be achieved. ${ }^{17}$

\section{Who should take care of patients with complex CHD?}

Does it always have to be a Paediatric Cardiac Anaesthesiologist? Or are there situations where a well prepared General Paediatric Anaesthesiologist might be the better choice, for complex neurosurgical or orthopaedic procedures, for example?

There are many different coverage models, depending on the institution, the patient and the case. In some hospitals, all cardiac cases are covered by Paediatric Cardiac Anaesthesiologists, in others only selective cases. Some places use a dedicated team of general paediatric anaesthesiologist to ensure adequate exposure. At Boston Children's, a member of the Cardiac Anaesthesia Division can be consulted and help with the planning, make recommendations or lend an additional pair of hands during the case. In smaller institutions, general paediatric anaesthesiologists or sometimes adult cardiac anaesthesiologists with paediatric experience cover these cases.

There is no evidence that one system is better than the others, but there is one interesting study looking at anaesthesiarelated cardiac arrests and system-related factors. After all risk adjustments for ASA status, age and setting, a low number of annual days delivering anaesthesia was associated with increased risk for cardiac arrests. ${ }^{18}$

\section{What/How can we prepare for the future?}

Several steps are necessary to master these challenges:

- The development and maintenance of well-defined networks of specialised centres and local hospitals with good communication and transfer policies. 
- More evidence-based standards and guidelines as well as multi-institutional research and cooperation.

- And above all, education and ongoing training of current and future paediatric and cardiac subspecialists but also general anaesthesiologists, surgeons and $\mathrm{OB}$ specialists.

- Everyone must be aware of the increased risk and the need for careful planning.

\section{References:}

1. Ramamoorthy C, Haberkern CM, Bhananker SM, et al. Anaesthesia-related cardiac arrest in children with heart disease: data from the Paediatric Perioperative Cardiac Arrest (POCA) registry. Anaesth Analg. 2010;110(5):137682. https://doi.org/10.1213/ANE.0b013e3181c9f927.

2. Van der Griend BF, Lister NA, McKenzie IM, et al. Postoperative mortality in children after 101,885 anaesthetics at a tertiary paediatric hospital. Anaesth Analg. 2011;112(6):1440-7. https://doi.org/10.1213/ANE.0b013e318213be52.

3. Sulkowski JP, Cooper JN, McConnell Pl, et al. Variability in noncardiac surgical procedures in children with congenital heart disease. J Pediatr Surg. 2014;49(11):1564-9. https://doi.org/10.1016/j.jpedsurg.2014.06.001.

4. Faraoni $D$, Zurakowski $D$, Vo $D$, et al. Post-operative outcomes in children with and without congenital heart disease undergoing noncardiac surgery. J Am Coll Cardiol. 2016;67(7):793-801. https://doi.org/10.1016/j.jacc.2015.11.057.

5. Chu DI, Tan JM, Mattei P, et al. Mortality and morbidity after laparoscopic surgery in children with and without congenital heart disease. J Pediatr. 2017;185:88-93. e3. https://doi.org/10.1016/j.jpeds.2017.02.011.

6. Chu DI, Tan JM, Mattei $P$, et al. Outcomes of laparoscopic and open surgery in children with and without congenital heart disease. J Pediatr Surg. 2018;53(10):1980-8. https://doi.org/10.1016/j.jpedsurg.2017.10.052.

7. Yuki K, Lee S, Staffa SJ, DiNardo JA. Induction techniques for pediatric patients with congenital heart disease undergoing noncardiac procedures are influenced by cardiac functional status and residual lesion burden. J Clin Anesth. 2018;50:14-7. https://doi.org/10.1016/j.jclinane.2018.06.022.
8. Faraoni D, Nasr VG, DiNardo JA. Overall hospital cost estimates in children with congenital heart disease: analysis of the 2012 Kid's Inpatient Database. Pediatr Cardiol. 2016;37:37-43. https://doi.org/10.1007/s00246-015-1235-0.

9. Christensen RE, Gholami AS, Reynolds PI, Malviya S. Anaesthetic management and outcomes after noncardiac surgery in patients with hypoplastic left heart syndrome: a retrospective review. Eur J Anaesthesiol. 2012;29(9):425-30. https:// doi.org/10.1097/EJA.0b013e328355345a.

10. Watkins S, Morrow SE, McNew BS, Donahue BS. Perioperative management of infants undergoing fundoplication and gastrostomy after stage I palliation of hypoplastic left heart syndrome. Pediatr Cardiol. 2012;33:697-704. https://doi org/10.1007/s00246-012-0197-8.

11. Brown ML, DiNardo JA, Odegard KC. Patients with single ventricle physiology undergoing noncardiac surgery are at high risk for adverse events. Paediatr Anaesth. 2015;25(8):846-51. https://doi.org/10.1111/pan.12685.

12. Jenkins KJ. Risk adjustment for congenital heart surgery: the RACHS-1 method. Semin Thorac Cardiovasc Surg Pediatr Card Surg Annu. 2004;7(1):180-4. https:// doi.org/10.1053/j.pcsu.2004.02.009.

13. White MC, Peyton J. Anaesthetic management of children with congenital heart disease for non-cardiac surgery. Contin Educ Anaesth Crit Care Pain. 2012;12(1):17-22. https://doi.org/10.1093/bjaceaccp/mkr049.

14. Faraoni D, Vo D, Nasr VG, DiNardo JA. Development and validation of a risk stratification score for children with congenital heart disease undergoing noncardiac surgery. Anesth Analg. 2016;123(4):824-30. https://doi.org/10.1213/ ANE. 0000000000001500 .

15. Brooks Peterson M, Houck CS, Deshpande JK, Flick RP. American College of Surgeons Children's surgery verification quality improvement program: what anesthesiologists need to know now. Anesth Analg. 2018;126(5):1624-32. https://doi.org/10.1213/ANE.0000000000002672.

16. DiNardo JA, Davis PJ. The American College of Surgeons Children's surgery verification quality improvement program: be careful what you wish for! Anesth Analg. 2018;126(5):1438-40. https://doi.org/10.1213/ANE.0000000000002810.

17. $\mathrm{Ng} \mathrm{SM}$, Jin $X$, Yates R, Kelsall AW. Outcome of noncardiac surgery in children with congenital heart disease performed outside a cardiac center. J Pediatr Surg. 2016;51(2):252-6. https://doi.org/10.1016/j.jpedsurg.2015.10.066.

18. Zgleszewski SE, Graham DA, Hickey PR, et al. Anesthesiologist- and system-related risk factors for risk-adjusted pediatric anesthesia-related cardiac arrest. Anesth Analg. 2016;122(2):482-9. https://doi.org/10.1213/ ANE.0000000000001059. 